\begin{tabular}{|c|c|c|}
\hline & Int.J.Curr.Microbiol.App.Sci (2021) 10(10): 21-35 & \\
\hline & $\begin{array}{l}\text { International Journal of Current Microbiology and Applied Sciences } \\
\text { ISSN: 2319-7706 Volume } 10 \text { Number } \mathbf{1 0}(\mathbf{2 0 2 1 )} \\
\text { Journal homepage: } \underline{\text { http://www.ijcmas.com }}\end{array}$ & 30 \\
\hline $\begin{array}{l}\text { EXCELLENT } \\
\text { PUBLISHERS }\end{array}$ & & \\
\hline
\end{tabular}

\title{
Bioanalytical Method Development and Validation of Dapoxetine Hydrochloride in Human Plasma by RP-HPLC
}

\author{
Pranit. B. Kale ${ }^{1 *}$, Santosh A. Waghmare ${ }^{1}$, Arun. M. Kashid ${ }^{2}$ and S. B. Wankhede ${ }^{3}$ \\ ${ }^{1}$ SWGP's Loknete Shri Dadapatil Pharate College of Pharmacy, Mandavgan Pharata, \\ Pune, Maharashtra- 412211, India \\ ${ }^{2}$ Sinhgad Institute of Pharmacy, Narhe, Pune-411 041, India \\ ${ }^{3}$ JSPM's Charak College of Pharmacy and Research, Wagholi, Pune-412 207, India \\ *Corresponding author
}

\section{A B S T R A C T}

Keywords

Bioanalytical method, Dapoxetin Hydrochloride, Human plasma, RPHPLC

Article Info

Accepted:

05 September 2021

Available Online:

10 October 2021
A simple, accurate and rapid Bioanalytical reverse phase high performance liquid chromatography (RPHPLC) method for determination of Dapoxetin hydrochloride in human plasma was validated as per ICH guideline. Dapoxetin hydrochloride is significantly superior in premature ejaculation and more active against serotonin transport inhibitor than any other drug in class. The total analysis was carried out on using stationary phase symmetry C1 $(4.6 \mathrm{~mm}$ X $250 \mathrm{~mm}, 5 \mu \mathrm{m})$ with Mobile Phase Acetonitrile: Buffer (60:40) $\mathrm{pH}$ adjusted to3.5 flow rate was $1.0 \mathrm{ml} / \mathrm{min}$, injection volume of $10 \mathrm{ppm}$ and detection wavelength was $293 \mathrm{~nm}$ at ambient temperature with total run time of 10 minutes. Retention time of spiked plasma and dapoxetine hydrochloride were found to be $2.153 \mathrm{~min}$ and $4.442 \mathrm{~min}, \mathrm{r} 2$ value were 0.995 and 0.999 and linearity range was $5 \mathrm{ppm}$ to $25 \mathrm{ppm}$ for both. The method was developed for accuracy, linearity, precision, recovery and stability in complies and stability in complies with CDER and ICH guideline.

\section{Introduction}

Dapoxetine is chemically (S)-N,N-dimetyl-3(naphtalen-1-yloxy)-1-phenylpropan-1-amine. Dapoxetine works by inhibiting the serotonin transporter, increasing serotonin's action at the post synaptic cleft, and as a consequence promoting ejaculatory delay. It is a medication used for the treatment of premature ejaculation (PE) in men 18-64 years old $(1,2,3$.

Dapoxetine is a selective serotonin reuptake inhibitor currently undergoing trials through Alza (under license from GenuPro, a 
collaboration between Eli Lilly and PPD). Dapoxetine is a short-acting SSRI drug currently being considered for approval by the Food and Drug Administration (FDA) for the treatment of premature ejaculation in men, which would make it the first drug approved for such treatment. Despite two clinical trials finished in 2006, experts doubt it will be approved by the FDA soon because SSRIs come with undesirable side-effects after longterm use, such as psychiatric problems, dermatological reactions, increase in body weight, lower sex-drive, nausea, headache, upset stomach and weakness, thus not significantly outweighing the benefit of premature ejaculation medication versus the risks $(3,4,5,6)$. By contrast with SSRIs approved for depression, which take 2 weeks or longer to reach steady-state concentration, dapoxetine has a unique pharmacokinetic profile, with a short time to maximum serum concentration (about $1 \mathrm{~h}$ ) and rapid elimination (initial half-life of 1-2 h). (Figure 1 Represent the Structure of Dapoxetine Hydrochloride).

\section{Materials and Methods}

Acetonitrile (HPLC Grade), Orthophosphoric acid (AR Grade), Potassium dihydrogen orthophosphate (Merck, AR Grade), Water for HPLC, Methanol (HPLC Grade) etc.

\section{Preliminary Analysis of dapoxetine $\mathrm{HCl}$}

Preliminary analysis of dapoxetine $\mathrm{HCl}$ such as description, solubility, and UV confirms the identification of dapoxetine $\mathrm{HCl}$ as per available literature.

\section{Solubility study}

Solubility of the drug was carried out with the different solvents such as methanol, acetonitrile, ethanol, $0.1 \mathrm{~N}$ hydrochloric acid, water and UV analysis was carried out to confirm the detection wavelength. The solution which shows the maximum absorbance was selected. Acetonitrile showed the maximum absorption at $292 \mathrm{~nm}$ wavelength.

\section{Selection of analytical wave length}

Name of drug : Dapoxetine $\mathrm{HCl}$

Concentration : $10 \mu \mathrm{g} / \mathrm{ml}$

Solvents : Buffer : Acetonitrile

Scanned range : 200-400 nm

Max Absorbance : $292.3 \mathrm{~nm}$

The maximum UV absorbance of dapoxetine $\mathrm{HCl}$ was found at $292.3 \mathrm{~nm}$. It was also observed that maximum absorption is occurs between 200-216 $\mathrm{nm}$ but at these wavelength sample matrix also absorbs significantly in this region.

\section{Analytical Method Development}

The analytical method has been developed after several permutations and combinations of mobile phase with stationary phase.

Table 1 represents the summary of several mobile phase compositions tried to optimize appropriate mobile phase composition for study. Various combinations showed peak asymmetry, theoretical plate less than 2000 and less retention time. Mobile phase consisting, acetonitrile: ammonium formate buffer $\mathrm{pH}-3.5$ in the ratio of $6: 4 \mathrm{v} / \mathrm{v}$ was selected as optimized mobile phase as it gave appropriate peak symmetry, theoretical plates and retention time.

\section{Optimized chromatographic conditions}

Summary of chromatographic parameters selected 
Column : Symmetry C18, $3.5 \mu \mathrm{m}, 250 \mathrm{~mm} \mathrm{x}$ $4.6 \mathrm{~mm}$

Mobile phase : Acetonitrile: Ammonium formate $(6: 4 \mathrm{v} / \mathrm{v})$

Flow rate : $1 \mathrm{ml} / \mathrm{min}$

Detection wavelength : $292 \mathrm{~nm}$

Sample injector : $20 \mu 1$ loop

Temperature : Ambient

Run time : 10 Min

\section{Bioanalytical Method validation}

\section{System suitability parameters}

\section{Results and Discussion}

It was found that the all system suitability parameters are within the acceptance criteria and method is suitable for determination of dapoxetine $\mathrm{HCl}$ from human plasma sample (Table 2.).

\section{Selectivity}

For selectivity, the blank samples of the plasma were obtained from six different persons. Each blank sample was tested for interference in dapoxetine peak.

The plasma and dapoxetine peak were well resolved. Typical chromatogram of blank human plasma and spiked in human plasma are shown in Fig. 5 and 6. It was found that peak from blank plasma does not interfere with peak of dapoxetine. Hence developed method is selective and peak obtained at 4.4 is only because of dapoxetine.

\section{Linearity and Range}

Linearity range of dapoxetine was observed at $5,10,15,20$ and $25 \mu \mathrm{g} / \mathrm{ml}$. The equation of linearity for dapoxetine was $\mathrm{y}=21742 \mathrm{x}+$ 69256. For all curves the correlation coefficient $\left(\mathrm{r}^{2}\right)$ is more than 0.999 . (Figure 7) The linearity range was observed in between $25-150 \mu \mathrm{g} / \mathrm{ml}$. (Table 3). For spiked human plasma Linearity range of dapoxetine was observed at 5, 10, 15, 20 and $25 \mu \mathrm{g} / \mathrm{ml}$. (Figure 8) The equation of linearity for dapoxetine was $\mathrm{y}=13601 \mathrm{x}+77162$. For all curves the correlation coefficient $\left(\mathrm{r}^{2}\right)$ was 0.995 (Table 4).

\section{Accuracy}

It can observe that all the obtained results of accuracy were satisfactory. The $\%$ mean accuracy lies between 88.00 - $106.00 \%$. Hence a good accuracy was observed by this method (Table 5, 6, 7).

\section{Precision}

It was found that the precision results were found satisfactory with respect to percent coefficient of variation $(\% \mathrm{CV})$ for all levels which were within the limit. The $\%$ coefficient of variation $(\% \mathrm{CV})$ of LQC sample is $1.31 \%$ and $\mathrm{t}$ he $\% \mathrm{CV}$ of MQC and HQC samples are 1.18 and $0.70 \%$ respectively. The developed method was précised for estimation of dapoxetine from human plasma. [Table 8 (Interday precision result of dapoxetine) and table 9 (Intraday precision results of dapoxetine)]. 
Table.1 Different trial for selection of the mobile phase

\begin{tabular}{|l|c|c|c|}
\hline \multicolumn{1}{|c|}{ Mobile phase } & $\begin{array}{c}\text { Composition } \\
\text { Ratio }\end{array}$ & $\mathbf{p H}$ & Retention Time \\
\hline Phosphate buffer: ACN & $50: 50$ & 3.5 & $8 \mathrm{~min}$ \\
\hline Phosphate buffer: ACN & $40: 60$ & 3.5 & $6.5 \mathrm{~min}$ \\
\hline Phosphate buffer: ACN & $30: 70$ & 3.5 & $5.8 \mathrm{~min}$ \\
\hline Ammonium formate: ACN & $40: 60$ & 3.5 & $4.4 \mathrm{~min}$ \\
\hline Ammonium formate: ACN & $30: 70$ & 3.5 & $4.1 \mathrm{~min}$ \\
\hline Ammonium formate: ACN & $20: 80$ & 3.5 & $3.9 \mathrm{~min}$ \\
\hline Water: ACN & $40: 60$ & 3.5 & $10 \mathrm{~min}$ \\
\hline Water: ACN & $30: 70$ & 3.5 & $8 \mathrm{~min}$ \\
\hline
\end{tabular}

Table.2 System suitability parameters

\begin{tabular}{|c|c|c|c|}
\hline Sr.No. & Parameters & Dapoxetine & Limit \\
\hline $\mathbf{1}$ & Resolution & 4.4 & $>2$ \\
\hline $\mathbf{2}$ & Column efficiency & 6960 & $>2000$ \\
\hline $\mathbf{3}$ & Symmetry factor & 1.54 & $<2$ \\
\hline $\mathbf{4}$ & Capacity factor & 6.3 & $>2$ \\
\hline
\end{tabular}

Table.3 Linearity of dapoxetine (API)

\begin{tabular}{|c|c|c|}
\hline Sr.No. & Standard concentration $(\boldsymbol{\mu g} / \mathbf{m l})$ & Mean Area \\
\hline $\mathbf{1}$ & 5 & 183047 \\
\hline $\mathbf{2}$ & 10 & 283509 \\
\hline $\mathbf{3}$ & 15 & 391946 \\
\hline $\mathbf{4}$ & 20 & 500193 \\
\hline $\mathbf{5}$ & 25 & 618266 \\
\hline
\end{tabular}

Table.4 Linearity for dapoxetine $\mathrm{HCl}$ spiked in human plasma

\begin{tabular}{|c|c|c|}
\hline Sr.No. & $\begin{array}{c}\text { Concentration } \\
(\boldsymbol{\mu g} / \mathbf{m l})\end{array}$ & Peak Area \\
\hline $\mathbf{1}$ & 5 & 149500 \\
\hline $\mathbf{2}$ & 10 & 214593 \\
\hline $\mathbf{3}$ & 15 & 268979 \\
\hline $\mathbf{4}$ & 20 & 352025 \\
\hline $\mathbf{5}$ & 25 & 420820 \\
\hline
\end{tabular}


Table.5 Accuracy result of dapoxetine ( $8 \mu \mathrm{g} / \mathrm{ml})$ (LQC)

\begin{tabular}{|c|c|c|c|c|}
\hline Sr.No. & Conc. $(\boldsymbol{\mu g} / \mathbf{m l})$ & Area & \% recovery & $\begin{array}{c}\text { Recovered concentration } \\
(\boldsymbol{\mu g} / \mathbf{m l})\end{array}$ \\
\hline $\mathbf{1}$ & 8 & 194336 & 99.14 & 7.93 \\
\hline $\mathbf{2}$ & 8 & 185138 & 90.5 & 8.52 \\
\hline $\mathbf{3}$ & 8 & 202234 & 106.53 & 8.52 \\
\hline \multicolumn{2}{|c|}{ Mean } & & 7.89 \\
\hline \multicolumn{2}{|c|}{ SD CV } & & 0.64 \\
\hline & \% Accuracy & & 8.10 \\
\hline
\end{tabular}

Table.6 Accuracy result of dapoxetine (16 $\mu \mathrm{g} / \mathrm{ml})$ (MQC)

\begin{tabular}{|c|c|c|c|c|}
\hline $\begin{array}{c}\text { Sr. } \\
\text { No. }\end{array}$ & $\begin{array}{c}\text { Concentration } \\
(\boldsymbol{\mu g} / \mathbf{m l})\end{array}$ & Area & \% recovery & $\begin{array}{c}\text { Recovered concentration } \\
(\boldsymbol{\mu g} / \mathbf{m l})\end{array}$ \\
\hline $\mathbf{1}$ & 16 & 301600 & 99.74 & 15.95 \\
\hline $\mathbf{2}$ & 16 & 293857 & 96.12 & 15.38 \\
\hline $\mathbf{3}$ & 16 & 309416 & 103.4 & 16.54 \\
\hline \multicolumn{2}{|c|}{ Mean } & & 15.96 \\
\hline \multicolumn{2}{|c|}{ SD CV } & & 0.5822 \\
\hline & \% Accuracy & & 3.64 \\
\hline
\end{tabular}

Table.7 Accuracy result of dapoxetine $(24 \mu \mathrm{g} / \mathrm{ml})$ (HQC)

\begin{tabular}{|c|c|c|c|c|}
\hline $\begin{array}{l}\text { Sr. } \\
\text { No. }\end{array}$ & $\begin{array}{c}\text { Concentration } \\
(\mu \mathrm{g} / \mathrm{ml})\end{array}$ & Area & \% recovery & $\begin{array}{l}\text { Recovered concentration } \\
\qquad(\mu \mathrm{g} / \mathrm{ml})\end{array}$ \\
\hline 1 & 24 & 401806 & 97.74 & 23.45 \\
\hline 2 & 24 & 413976 & 101.54 & 24.37 \\
\hline 3 & 24 & 401980 & 94.11 & 22.58 \\
\hline \multicolumn{4}{|c|}{ Mean } & 23.47 \\
\hline \multicolumn{4}{|c|}{ SD } & 0.8913 \\
\hline \multicolumn{4}{|c|}{$\% \mathrm{CV}$} & 3.79 \\
\hline \multicolumn{4}{|c|}{ \% Accuracy } & 97.80 \\
\hline
\end{tabular}


Table.8 Interday precision result of dapoxetine

\begin{tabular}{|c|c|c|c|}
\hline \multirow[t]{2}{*}{$\mathbf{A}$} & DAY 1 & DAY 2 & DAY 3 \\
\hline & \multicolumn{3}{|c|}{ Concentration 8 g/mL (LQC) } \\
\hline 1 & 194536 & 187807 & 189987 \\
\hline 2 & 189145 & 190987 & 184928 \\
\hline 3 & 192245 & 189234 & 183025 \\
\hline 4 & 186087 & 188086 & 192851 \\
\hline 5 & 179987 & 179987 & 190167 \\
\hline 6 & 187895 & 185453 & 188042 \\
\hline Mean & 188282 & 186925 & 188166 \\
\hline SD & 5037.3 & 3853 & 363.65 \\
\hline$\% \mathrm{CV}$ & 2.675 & 2.061 & $\mathbf{1 . 9 3 3 7}$ \\
\hline B & \multicolumn{3}{|c|}{ Concentration 16 g/mL(MQC) } \\
\hline 1 & 301600 & 301587 & 298754 \\
\hline 2 & 294876 & 295776 & 294536 \\
\hline 3 & 298987 & 300123 & 294536 \\
\hline 4 & 293334 & 298347 & 294332 \\
\hline 5 & 295112 & 294434 & 301165 \\
\hline 6 & 293223 & 295334 & 293564 \\
\hline Mean & 296188 & 297600 & 297392 \\
\hline SD & 3374 & 2874 & 3728 \\
\hline$\% \mathrm{CV}$ & 1.139 & 0.965 & 1.25 \\
\hline $\mathbf{C}$ & \multicolumn{3}{|c|}{ 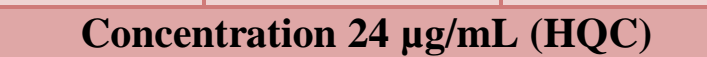 } \\
\hline 1 & 403112 & 401587 & 398754 \\
\hline 2 & 405876 & 410276 & 404536 \\
\hline 3 & 403223 & 409123 & 404332 \\
\hline 4 & 408987 & 408347 & 401165 \\
\hline 5 & 402334 & 404434 & 405422 \\
\hline 6 & 409112 & 406516 & 403564 \\
\hline Mean & 405440 & 405334 & 402962 \\
\hline SD & 3040 & 3295 & 2581 \\
\hline$\% \mathrm{CV}$ & 0.75 & 0.810 & 0.62 \\
\hline
\end{tabular}


Table.9 Intraday precision results of dapoxetine

\begin{tabular}{|c|c|c|c|}
\hline \multirow[t]{2}{*}{$\mathbf{A}$} & 1st reading & 2nd reading & 3rd reading \\
\hline & \multicolumn{3}{|c|}{ Concentration $8 \mu \mathrm{g} / \mathrm{mL}$ (LQC) } \\
\hline 1 & 194536 & 189452 & 187807 \\
\hline 2 & 189145 & 183816 & 190987 \\
\hline 3 & 192245 & 183259 & 189234 \\
\hline 4 & 186087 & 192851 & 188086 \\
\hline 5 & 179987 & 190579 & 179987 \\
\hline 6 & 187895 & 188042 & 185453 \\
\hline Mean & 188282 & 187999 & 186925 \\
\hline SD & $\mathbf{5 0 3 7 . 3}$ & 363.65 & 3853 \\
\hline$\% \mathrm{CV}$ & 2.801 & 1.631 & 2.061 \\
\hline B & \multicolumn{3}{|c|}{ Concentration $16 \mu \mathrm{g} / \mathrm{mL}(\mathrm{MQC})$} \\
\hline 1 & 301600 & 298025 & 301587 \\
\hline 2 & 294876 & 2940391 & 295776 \\
\hline 3 & 298987 & 294805 & 300123 \\
\hline 4 & 293334 & 294279 & 298347 \\
\hline 5 & 295112 & 301851 & 294434 \\
\hline 6 & 293223 & 293489 & 295334 \\
\hline Mean & 296188 & 296801 & 297600 \\
\hline SD & 3374 & 3728 & 2874 \\
\hline$\% \mathrm{CV}$ & 1.537 & 1.35 & 1.28 \\
\hline C & \multicolumn{3}{|c|}{ Concentration $24 \mu \mathrm{g} / \mathrm{mL}$ (HQC) } \\
\hline 1 & 403112 & 398754 & 401587 \\
\hline 2 & 405876 & 404536 & 410276 \\
\hline 3 & 403223 & 404332 & 409123 \\
\hline 4 & 408987 & 401165 & 408347 \\
\hline 5 & 402334 & 405422 & 404434 \\
\hline 6 & 409112 & 403564 & 406516 \\
\hline Mean & 405440 & 402962 & 405334 \\
\hline SD & 3040 & 2581 & 3295 \\
\hline$\% \mathrm{CV}$ & 0.91 & 0.71 & 0.85 \\
\hline
\end{tabular}

Table.10 Recovery results of dapoxetine $(8 \mu \mathrm{g} / \mathrm{ml})$ (LQC)

\begin{tabular}{|c|c|c|}
\hline Replicate & $\begin{array}{c}\text { Peak Area of Standard } \\
\text { drug }\end{array}$ & $\begin{array}{c}\text { Peak area of drug Spiked in } \\
\text { plasma }\end{array}$ \\
\hline $\mathbf{1}$ & 243509 & 194336 \\
\hline $\mathbf{2}$ & 250012 & 204136 \\
\hline $\mathbf{3}$ & 249221 & 195554 \\
\hline Mean & 247580 & 198008 \\
\hline SD & 3548 & 5341 \\
\hline \% CV & 1.43 & 2.69 \\
\hline \% Mean Recovery & & \\
\hline
\end{tabular}


Table.11 Recovery results of dapoxetine $(16 \mu \mathrm{g} / \mathrm{ml})$ (MQC)

\begin{tabular}{|c|c|c|}
\hline Replicate & $\begin{array}{c}\text { Peak Area of } \\
\text { Standard drug }\end{array}$ & $\begin{array}{c}\text { Peak area of drug Spiked in } \\
\text { plasma }\end{array}$ \\
\hline $\mathbf{1}$ & 421946 & 301600 \\
\hline $\mathbf{2}$ & 419963 & 298895 \\
\hline $\mathbf{3}$ & 422015 & 301945 \\
\hline Mean & 421308 & 300813 \\
\hline SD & 1165 & 1670 \\
\hline \% CV & 0.27 & 0.55 \\
\hline
\end{tabular}

Table.12 Recovery results of dapoxetine $(24 \mu \mathrm{g} / \mathrm{ml})$ (HQC)

\begin{tabular}{|c|c|c|}
\hline Replicate & Peak Area of Standard drug & Peak Area of spiked plasma drug \\
\hline $\mathbf{1}$ & 598222 & 401806 \\
\hline $\mathbf{2}$ & 594256 & 405345 \\
\hline $\mathbf{3}$ & 589996 & 399321 \\
\hline Mean & 594158 & 402157 \\
\hline SD & 4113 & 3027 \\
\hline \% CV & 6.92 & 0.75 \\
Recovery & & \\
\hline
\end{tabular}

Table.13 Freeze and thaw stability result of dapoxetine $(8 \mu \mathrm{g} / \mathrm{ml})(\mathrm{LQC})$

\begin{tabular}{|c|c|c|}
\hline Replicate & Peak Area of Standard Sample & Peak Area of Stability Sample \\
\hline $\mathbf{1}$ & 7.89 & 7.01 \\
\hline $\mathbf{2}$ & 8.06 & 7.6 \\
\hline $\mathbf{3}$ & 8.1 & 7.4 \\
\hline Mean & 8.01 & 7.33 \\
\hline SD & 0.11 & 0.30 \\
\hline \% CV & 1.35 & 1.26 \\
\hline \% Mean & \multicolumn{2}{|}{} \\
\hline Stability & \multicolumn{2}{|c|}{} \\
\hline
\end{tabular}

Table.14 Freeze and thaw stability result of dapoxetine $(24 \mu \mathrm{g} / \mathrm{ml})(\mathrm{HQC})$

\begin{tabular}{|c|c|c|}
\hline Replicate & Peak Area of standard sample & Peak Area of stability sample \\
\hline $\mathbf{1}$ & 24.18 & 23.2 \\
\hline $\mathbf{2}$ & 24.64 & 23.01 \\
\hline $\mathbf{3}$ & 23.52 & 22.5 \\
\hline Mean & 24.11 & 22.90 \\
\hline SD & 0.562 & 0.361 \\
\hline \% CV & 2.33 & 1.57 \\
\hline
\end{tabular}


Table.15 Short-term stability results of dapoxetine $(8 \mu \mathrm{g} / \mathrm{ml})(\mathrm{LQC})$

\begin{tabular}{|c|c|c|}
\hline Replicate & $\begin{array}{c}\text { Peak Area of } \\
\text { Standard sample }\end{array}$ & $\begin{array}{c}\text { Peak Area of } \\
\text { Stability sample }\end{array}$ \\
\hline $\mathbf{1}$ & 8.23 & 7.8 \\
\hline $\mathbf{2}$ & 8.06 & 7.4 \\
\hline $\mathbf{3}$ & 8.1 & 7.2 \\
\hline Mean & 8.13 & 7.46 \\
\hline SD & 0.08 & 0.30 \\
\hline \% CV & 1.08 & 1.30 \\
\hline \% Mean Stability & \multicolumn{2}{|c}{} \\
\hline
\end{tabular}

Table.16 Short-term stability result of dapoxetine ( $24 \mu \mathrm{g} / \mathrm{ml})(\mathrm{HQC})$

\begin{tabular}{|c|c|c|}
\hline Replicate & $\begin{array}{c}\text { Peak Area of } \\
\text { Standard sample }\end{array}$ & $\begin{array}{c}\text { Peak Area of } \\
\text { Stability sample }\end{array}$ \\
\hline $\mathbf{1}$ & 24.01 & 22.01 \\
\hline $\mathbf{2}$ & 24.23 & 22.98 \\
\hline $\mathbf{3}$ & 23.96 & 22.45 \\
\hline Mean & 24.06 & 22.48 \\
\hline SD & 0.143 & 0.48 \\
\hline \% CV & 0.59 & 2.13 \\
\hline \% Mean Stability & $93.66 \%$ & \\
\hline
\end{tabular}

Table.17 Long-term stability result of dapoxetine ( $8 \mu \mathrm{g} / \mathrm{ml})$ (LQC)

\begin{tabular}{|c|c|c|}
\hline Replicate & $\begin{array}{c}\text { Peak area of } \\
\text { standard sample }\end{array}$ & $\begin{array}{c}\text { Peak area of } \\
\text { Stability sample }\end{array}$ \\
\hline $\mathbf{1}$ & 7.98 & 6.85 \\
\hline $\mathbf{2}$ & 8.1 & 6.99 \\
\hline $\mathbf{3}$ & 8.2 & 7.02 \\
\hline Mean & 8.09 & 6.95 \\
\hline SD & 0.11 & 0.09 \\
\hline \% CV & 1.35 & 1.29 \\
\hline \% Mean Stability & & $85.9 \%$ \\
\hline
\end{tabular}

Table.18 Long-term stability result of dapoxetine (24 $\mu \mathrm{g} / \mathrm{ml})(\mathrm{HQC})$

\begin{tabular}{|c|c|c|}
\hline Replicate & $\begin{array}{c}\text { Peak Area of standard } \\
\text { sample }\end{array}$ & $\begin{array}{c}\text { Peak area of } \\
\text { Stability sample }\end{array}$ \\
\hline $\mathbf{1}$ & 24.02 & 21.04 \\
\hline $\mathbf{2}$ & 24.12 & 21.2 \\
\hline $\mathbf{3}$ & 24.2 & 21.5 \\
\hline Mean & 24.11 & 21.14 \\
\hline SD & 0.090 & 0.092 \\
\hline \% CV & 0.37 & 0.42 \\
\hline \% Mean Stability & \multicolumn{2}{|c}{} \\
\hline
\end{tabular}


Table.19 Stock solution stability result of dapoxetine $(8 \mu \mathrm{g} / \mathrm{ml})$ (LQC)

\begin{tabular}{|c|c|c|}
\hline Replicate & Peak area of standard sample & Peak area of stability sample \\
\hline $\mathbf{1}$ & 8.12 & 7.24 \\
\hline $\mathbf{2}$ & 8.1 & 7.12 \\
\hline $\mathbf{3}$ & 7.86 & 7.01 \\
\hline Mean & 8.02 & 7.12 \\
\hline SD & 0.144 & 0.11 \\
\hline \% CV & 1.80 & 1.61 \\
\hline
\end{tabular}

Table.20 Stock solution stability result of dapoxetine $(24 \mu \mathrm{g} / \mathrm{ml})(\mathrm{HQC})$

\begin{tabular}{|c|c|c|}
\hline Replicate & Peak area of standard sample & Peak area of stability sample \\
\hline $\mathbf{1}$ & 24.2 & 21.56 \\
\hline $\mathbf{2}$ & 23.54 & 21.01 \\
\hline $\mathbf{3}$ & 23.98 & 21.4 \\
\hline Mean & 23.76 & 21.32 \\
\hline SD & 0.38 & 0.282 \\
\hline \% CV & 1.60 & 1.326 \\
\hline
\end{tabular}

Table.21 Summary of all validation parameter results

\begin{tabular}{|c|c|c|}
\hline Sr. No & Validation Parameters & Result \\
\hline $\mathbf{1}$ & Linearity range $(\mu \mathrm{g} / \mathrm{ml})$ & $5-25$ \\
\hline $\mathbf{2}$ & Retention time $(\mathrm{min})$ & 4.4 \\
\hline $\mathbf{3}$ & Correlation coefficient $(\mathrm{r} 2)$ & 0.995 \\
\hline $\mathbf{4}$ & Intraday Precision $($ mean \%CV) & 7.327 \\
\hline $\mathbf{5}$ & Inter day Precision $($ mean \%CV) & 6.26 \\
\hline $\mathbf{6}$ & Mean Recovery $(\%)$ & 73.01 \\
\hline $\mathbf{7}$ & Freeze and Thaw stability \% Mean stability & 93.27 \\
\hline $\mathbf{8}$ & Short-term temperature stability \% Mean stability & 92.70 \\
\hline $\mathbf{9}$ & Long-term stability \% Mean stability & 87.34 \\
\hline $\mathbf{1 0}$ & Stock solution stability \% Mean stability & 89.24 \\
\hline
\end{tabular}

Fig.1 Structure of Dapoxetine<smiles>CN(C)[C@@H](CCOC1C=CC=C2C=CC=CC21)c1ccccc1</smiles> 
Int.J.Curr.Microbiol.App.Sci (2021) 10(10): 21-35

Fig.2 UV spectrum of dapoxetine HCL

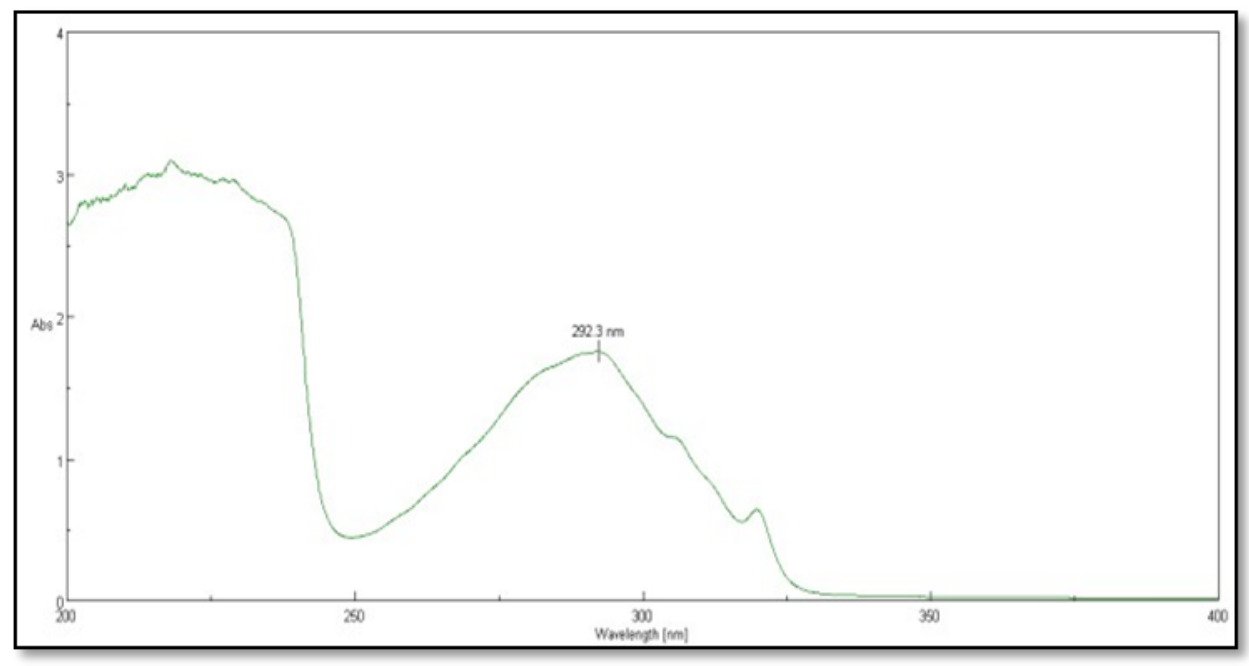

Fig.3 HPLC Chromatogram of Dapoxetine HCL $(15 \mu \mathrm{g} / \mathrm{ml})$

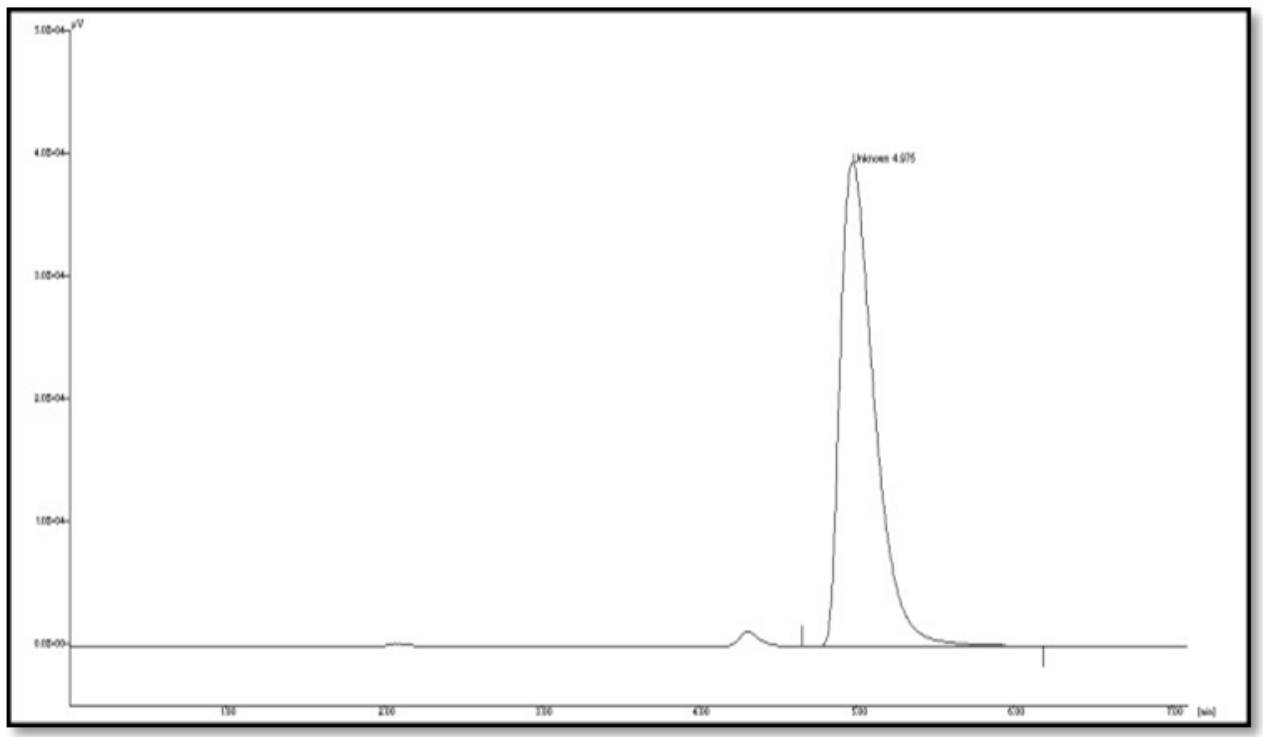


Fig.4 Chromatogram of Dapoxetine HCL Spiked in Human Plasma $(10 \mu \mathrm{g} / \mathrm{ml})$

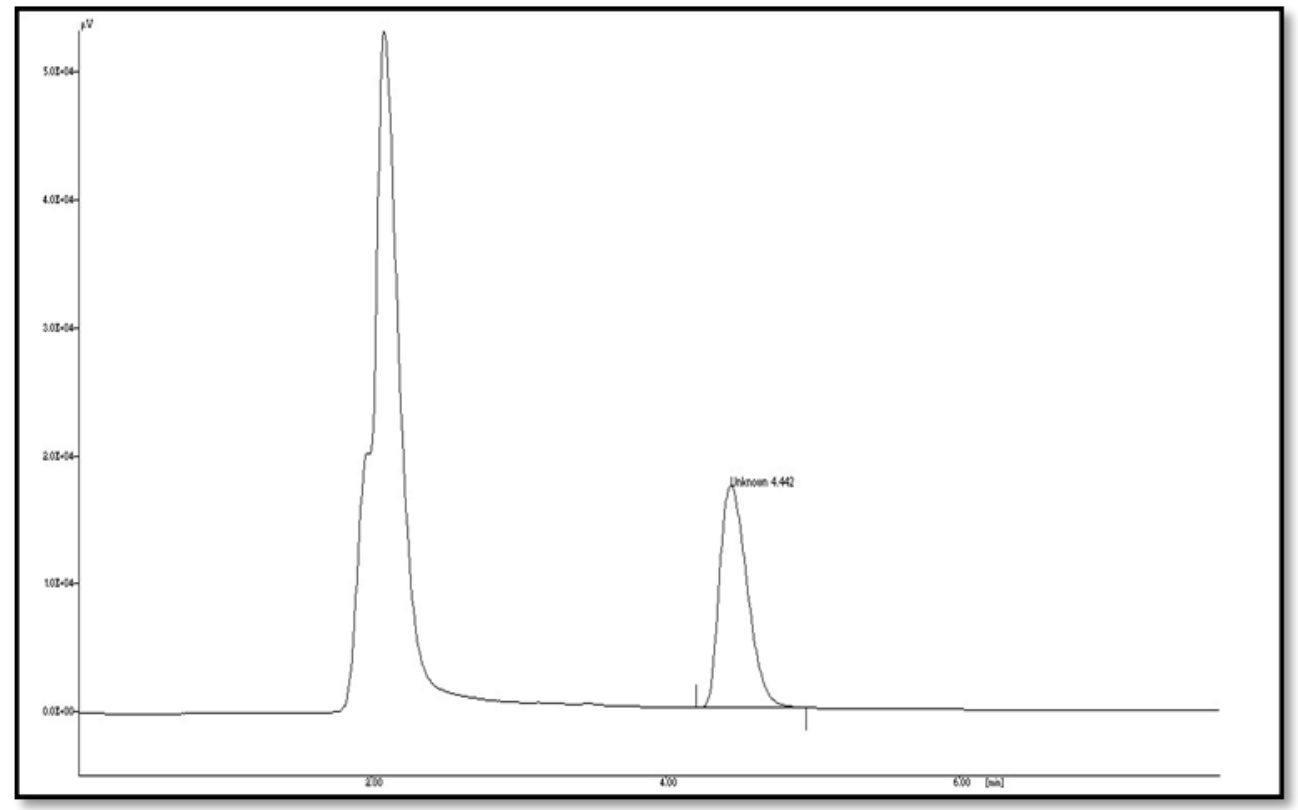

Fig.5 A Typical Chromatogram of Blank Human Plasma

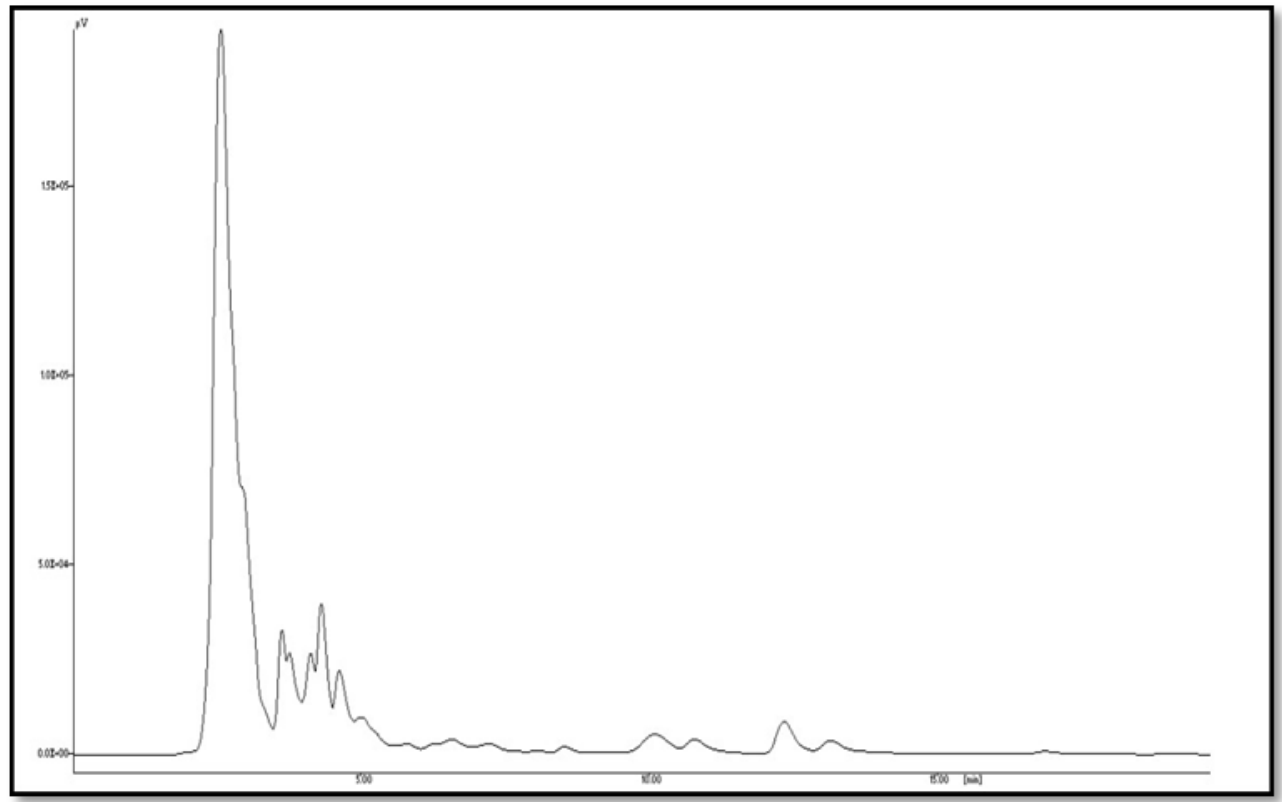


Fig.6 Typical Chromatogram of Dapoxetine

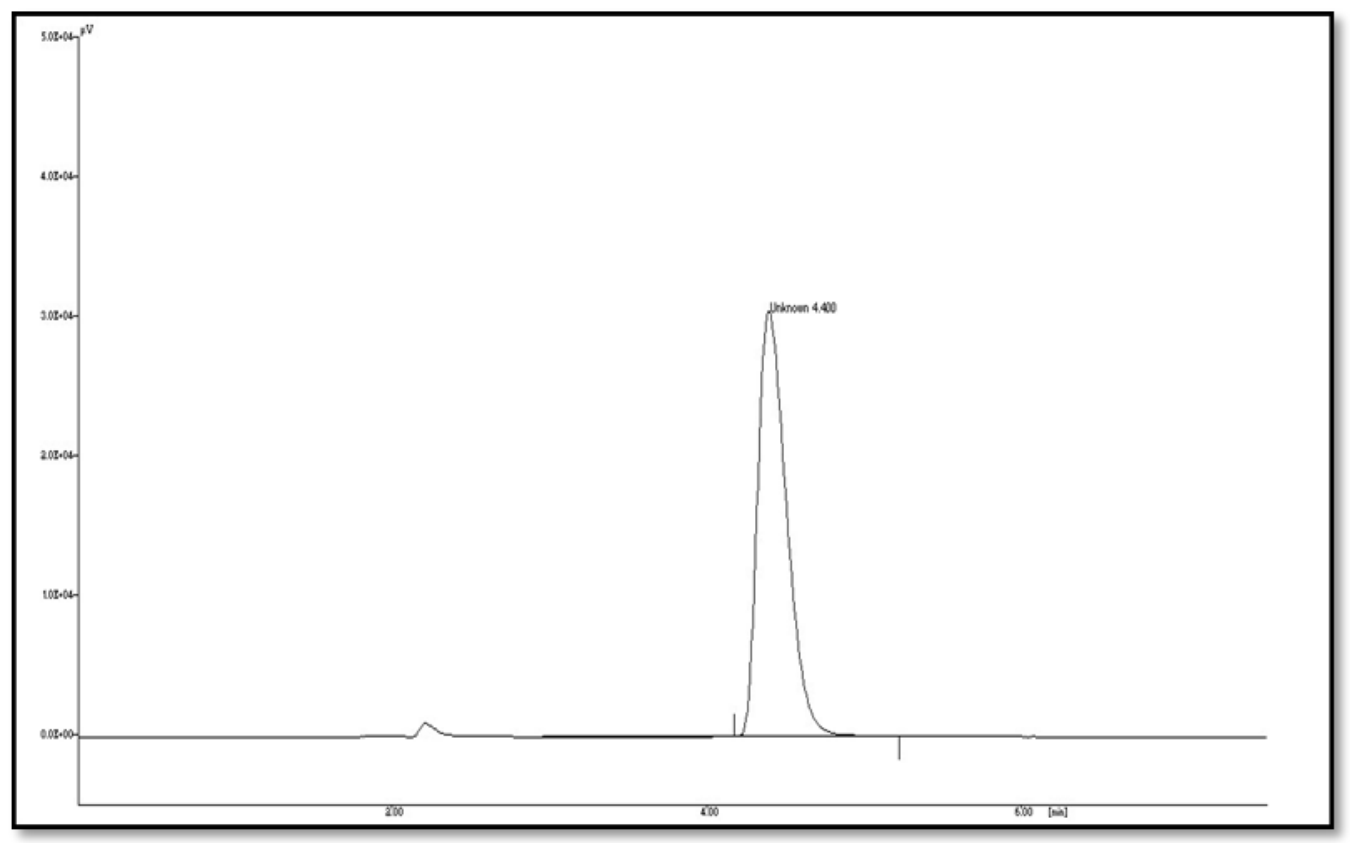

Fig.7 Calibration Curve for Dapoxetine (API)

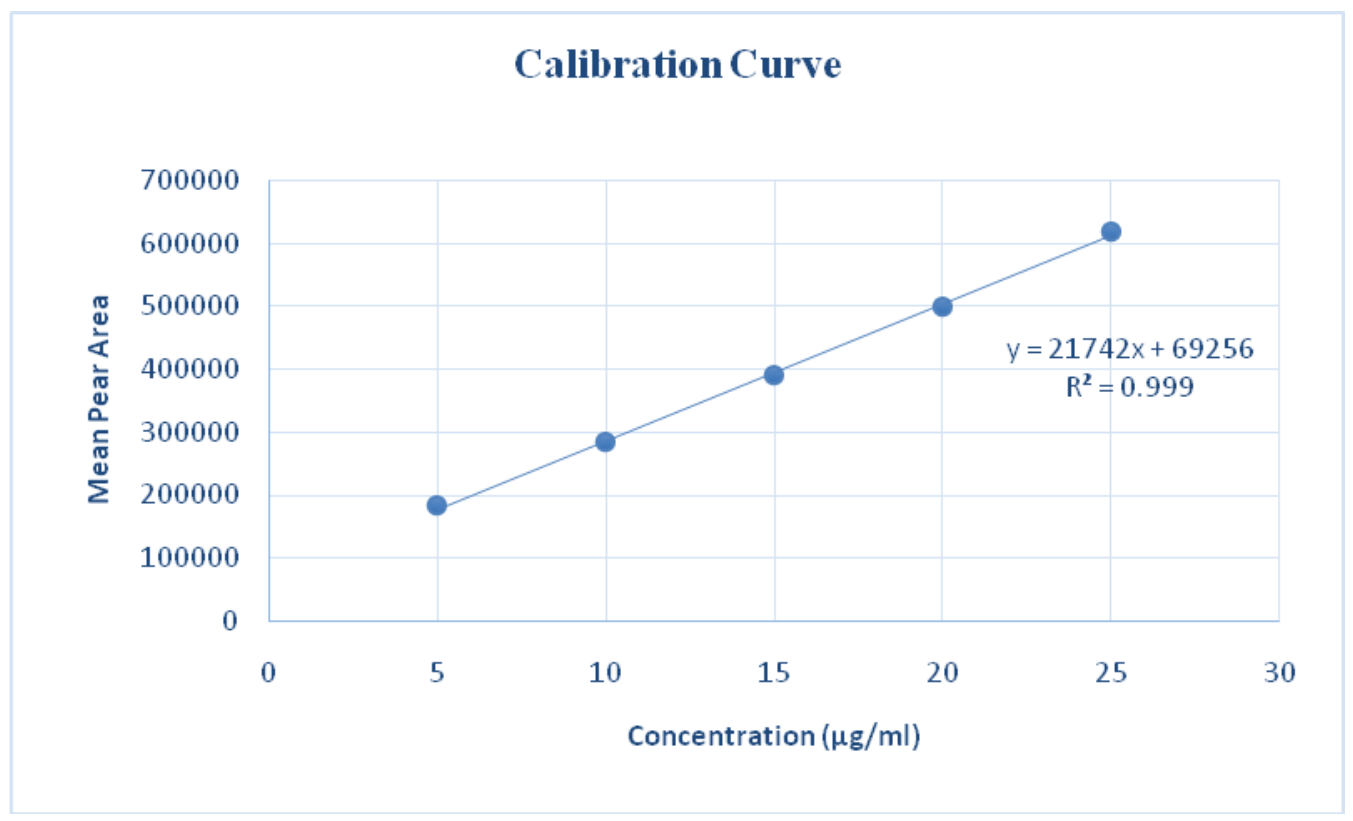


Fig.8 Calibration Curve of Dapoxetine Spiked in Human Plasma

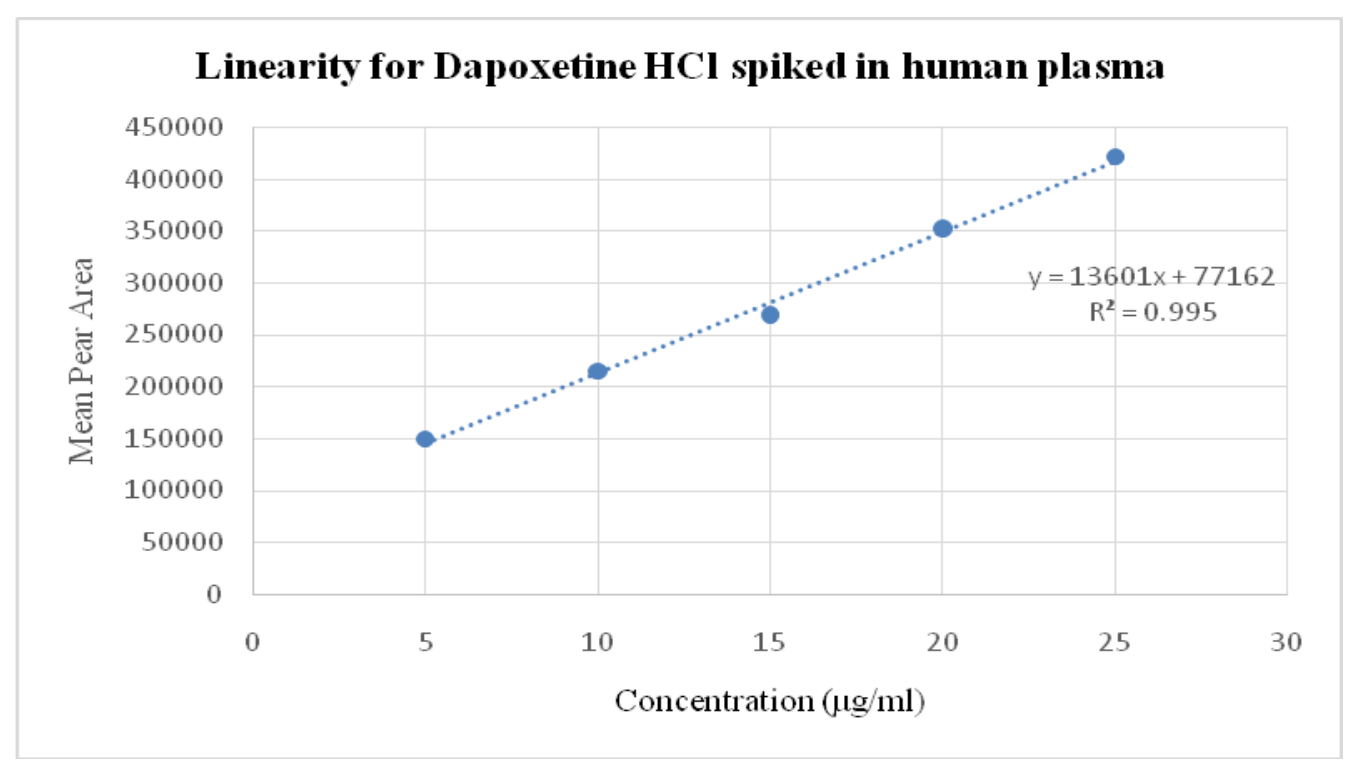

\section{Recovery}

The percent recovery of dapoxetine from human plasma was found to be 97.97, 99.39, 98.68 for LQC, MQC and HQC level. The recovery of dapoxetine ease consistent, precise and reproducible (Table 10, 11 and 12).

\section{Stability}

\section{Freeze and Thawstability}

Dapoxetine did not show significant alteration in its concentration even after three cycles of freeze and thaw (Table 13 and 14).

\section{Short-term temperature stability}

According to observed results the concentration of dapoxetine in the biological sample did not show significant alteration in its concentration. Samples are stable up to 6 hrs (Table 15 and 16).

\section{Long-term stability}

The percent mean long-term temperature stability of dapoxetine in the biological sample did not show significant alteration in its concentration even after 7 days. After 21 days the concentration of dapoxetine decreased up to $30 \%$ within the time period under the indicated storage conditions, however the days (Table 17 and 18).

\section{Stock solution stability}

Dapoxetine when stored at room temperature did not show significant alteration in its concentration; hence the standard stock solution can be stored upto $6 \mathrm{hrs}$ after its preparation. Sample did not show significant alteration in its concentration after $6 \mathrm{hrs}$ (Table 19 and 20).

\section{Summary of all validation parameter results}

All the parameters of validation were found within the acceptance criteria as per USFDA guidelines. Thus, we conclude the developed bioanalytical method was suitable for determination of dapoxetine from human plasma and can be further applied in of pharmacokinetic study of dapoxetine (Table 21). 


\section{References}

1. Amin, G., Chapla, B., Pandya, A., Kakadiya, J., Baria, D. 2012. 'Development and validation of dual wavelength UVSpectrophotometric methods for simultaneous estimation of tadaafil and dapoxetine hydrochloride in their combined tablet dosage form', International Journal of Pharmaceutical Research and Bio-Science, Vol. 1, no. 2, pp. 247-255.

2. Ahuja, S., Rasmussen, H. 2007. 'HPLC Method Development for Pharmaceuticals', 1st edition. Academic Press,

3. Brahmankar, D. 1995. 'Biopharmaceutics and Pharmacokinetics-A Treatise', $2^{\text {nd }}$ edition. Vallabhprakashan, Delhi, India.

4. Beckett, A., Stenlake, J. 1997. 'Practical Pharmaceutical Chemistry', $4^{\text {th }}$ edition. Athlone Press, UK.

5. Chatwal, G., Anand. S., 2008. 'Instrumental Methods of Chemical Analysis', $4^{\text {th }}$ edition. Himalaya Publishing House, India.

6. Conners, K. 1982. 'A Textbook of Pharmaceutical Analysis', $3^{\text {rd }}$ edition. WileyInterscience Publication, United states.

7. List of approved drug by CDSCO available from:

URL: https://cdsco.nic.in/listofdrugapprovedmain.ht $\mathrm{ml}$

8. Product monograph of Dapoxetine published by emcure pharmaceuticals Ltd. Available from:

URL: www.sustinexprematureejaculation.com/pdf/ Sustin ex.pdf

9. Janssen-Cilag EMEA announces receipt of first regulatory approvals for Priligy for PE in Finland and Sweden. Available from: URL: www.investor.jnj.com/textonly/pressrelease.cfm

10. Australian public assessment report for
Dapoxetine published on 2010. by janssencilagpvt. Ltd. Available from: URL: www.tga.gov.au/pdf/auspar/auspar-

priligy.pdf L Hamilton, J David Cornpropst, Journal of Chromatography B: Biomedical Sciences and Applications, 612(2)(1993).

11. M R. Safarinejad. Safety and Efficacy of Dapoxetine in the Treatment of Premature Ejaculation: A Double-Blind, PlaceboControlled, Fixed-Dose, Randomized Study. Neuropsychopharmacology. 2007;33:1259.

12. Dresser M J, Desai D, Gidwani S, Seftel A D, Modi N B Dapoxetine, A novel treatment for premature ejaculation, does not have pharmacokinetic interactions with phosphodiesterase-5 inhibitors. International Journal of Impotence Research 2006; 18:10410.

13. Q2R1 ICH guidelines for analytical method development. Available at: https://www. ich.org/fileadmin/Public_Web_Site/IC

H_Products/Guidelines/Quality/Q2_R1/Step4 /Q2 _R1_Guideline.pdf

14. Kanani V V, Gohil V P, Muralikrishna K S, Deshmukh A, 2012. Design and validation of dissolution profile of Dapoxetine $\mathrm{HCl}$ by using spectrophotometric method in dosage form. Inventi Impact: Pharm Ana \&Qual Assur, 1.

15. Breier A R, Paim C S, Stepe M, Schapoval E E S, J Pharm PharmSci, 8 (2005).

16. Kevin K.-C. Liu, Sakya S M, O'donnellCO, Flick A C, Jin Li. Synthetic approaches to the 2009. new drugs. Elsevier: bioorganic and medicinal chemistry. 2011;19:1136-54.

17. Silvia H, Borgmann M, Parcianello L, Arend M Z, Lizianebajerski, Simone G. Cardoso Development and validation of a Dissolution method with Spectrophotometric analysis for Diacerhein capsules. Scientiapharmaceutica. 2008;76(541-54).

\section{How to cite this article:}

Pranit. B. Kale, Santosh A. Waghmare, Arun. M. Kashid and Wankhede, S. B. 2021. Bioanalytical Method Development and Validation of Dapoxetine Hydrochloride in Human Plasma by RP-HPLC. Int.J.Curr.Microbiol.App.Sci. 10(10): 21-35.

doi: https://doi.org/10.20546/ijcmas.2021.1010.004 